\title{
Farmacologix 1.0 Alternativa pedagógica en el área de la farmacología clínica y terapéutica veterinaria
}

\section{Farmacologix 1.0 Educational alternative in the area of veterinary clinical pharmacology and therapeutics}

\author{
José L. Bothia ${ }^{1}$, Narciso J. Tolosa ${ }^{1}$, Audel J. Díaz², Dumar A. Jaramillo H. ${ }^{3}$ \\ ${ }^{1} \mathrm{MVZ}$, Universidad de los Llanos. ${ }^{2}$ Ingeniero de sistemas. y \\ ${ }^{3} \mathrm{MVZ}, \mathrm{MSc}(\mathrm{c})$, Docente Universidad de los Llanos \\ jlbothia@gmail.com
}

Recibido 09 Noviembre de 2009, aprobado 01 Diciembre 2010

\section{RESUMEN}

El objetivo de este trabajo fue elaborar un software (sistema de registro de datos de escritorio) educativo basado en el área de Farmacología Veterinaria. El proyecto surge de la necesidad de solucionar dificultades importantes relacionadas con la consecución de datos y veracidad de los mismos, dificultad atribuida a la complejidad de los elementos que componen esta área del conocimiento; la dispersión y falta de sistematización de información ya existente es una constante que origina los problemas básicos de la actividad de prescripción a nivel de campo y el estudio de la farmacología veterinaria. Se eligió implementar un sistema de información como un instrumento de ayuda educativa donde se centralizó y se creó un compendio de información de Farmacología Clínica y Terapéutica Veterinaria, partiendo del conocimiento contenido en fuentes bibliográficas, documentales y humanas, entre otras. Esta aplicación permite que las personas registradas como administrador realicen aportes a la base de datos, actualizaciones congruentes con el contexto y la evolución de las ciencias farmacológicas y las personas que no cuentan con este beneficio, puedan consultar esta información sin restricciones. El proceso de desarrollo utilizado para la generación de Farmacologix 1.0 sigue la metodología de programación extrema donde se utilizó la herramienta PostgreSQL versión 8.4 para Windows 32 
bits 1 de julio de 2009, publicado bajo la licencia BSD (Berkeley Software Distribution); dirigido por una comunidad de desarrolladores denominada el PGDG (PostgreSQL Global Development Group.), como gestor para las bases de datos y Java 18 en la generación del interfaz de presentación al usuario. Como resultado de la creación del software Farmacologix 1.0, se obtiene una presentación de la información de manera sencilla y ágil para los usuarios que se conecten a la red de área local del Laboratorio de Fisiología de la Universidad de los Llanos, en la cual se podrá obtener información contextualizada y centralizada de los fármacos de distribución nacional y regional con usos en la medicina veterinaria. Este software fue basado en el microdiseño del curso de Farmacología Veterinaria del Programa de Medicina Veterinaria y Zootecnia de la Universidad de los Llanos.

Palabras claves: Farmacología veterinaria, principio activo, sistema de registro de datos, software, tecnología de la información y las comunicaciones (TIC).

\section{ABSTRACT}

The aim of this study was to develop a software (data logging system desktop) based education in the area of Veterinary Pharmacology. The project stems from the need to solve important problems related to the implementation of information and veracity, difficulty attributed to the complexity of the elements that make up this area of knowledge dispersion and lack of systematization of existing information is a constant giving rise to the basic problems of limitation of activity at the field level and the study of veterinary pharmacology. It chose to implement an information system as an instrument of educational assistance which was centralized and created a compendium of information of Veterinary Clinical Pharmacology and Therapeutics, based on the knowledge contained in the available literature, documentaries and, amongst others. This application allows people registered as an administrator made contributions to the database updates the context and consistent with the development of pharmacological sciences and those who do not have this benefit, you can access this information 
without restrictions. The development process used to generate Farmacologix 1.0 follows the methodology of extreme programming where the tool was used PostgreSQL version 8.4 for Windows 32-bit July 1, 2009, published under the license BSD (Berkeley Software Distribution), led by a community of developers known as the PGDG (PostgreSQL Global Development Group.), as the manager for databases and Java 18 in the generation of the user interface presentation. As a result of the creation of Farmacologíx 1.0 software, you get a presentation information in a simple and quick for users to connect to local area network of the Laboratory of Physiology, in which contextual information can be obtained drugs and centralized national and regional distribution in Veterinary Medicine. This software was curriculum based on the area Veterinary Pharmacology of Program Veterinary Medicine and husbandry, Universidad de los Llanos.

Keywords: Active principles, data recording system, software, information technology and communication (ICT), veterinary pharmacology.

\section{INTRODUCCIÓN}

La demanda de información sobre diversos aspectos ha impulsado el desarrollo de registro de datos, los cuales nos suministran información que cubren diferentes áreas y satisfacen los requerimientos de una amplia gama de usuarios (Majó, 2003). En la actualidad se encuentra que el currículo académico de la Universidad de los Llanos cuenta dentro de sus estrategias pedagógicas con varias alternativas para la actividad de enseñanza-aprendizaje de los cursos, incluidas en su plan de estudios, como son las clases presenciales, las clases magistrales y la destinación de tiempo presencial y libre (créditos académicos), por parte del profesor y/o del discente, a la profundización de temas más específicos dependiendo del interés de la persona sobre el conocimiento de las Ciencias Veterinarias; esta profundización se realiza por medio de revisiones bibliográficas de literatura principalmente (Programa de Medicina veterinaria y 
Zootecnia, 2004), donde el principal inconveniente es la falta de documentación o de herramientas que centralicen y filtren la información.

En internet se encuentran fuentes bibliográficas referentes a información de farmacológica veterinaria, pero de manera parcializada a través de estudios científicos específicos y/o vademécum de casas comerciales que no aportan información científica detallada (APROVET, 2007; VECOL, 2003; SCHERINGPLOUGH S.A., 2004; P.R. Vademecum, 2009; ERMA, 2004) además que en Colombia existe muy poca información veraz en medio digital sobre la Farmacología Veterinaria, la oferta real de medicamentos y la posibilidad de uso de preparados comerciales para humanos. El objetivo de este trabajo fue desarrollar un software (sistema de registro de datos de escritorio) educativo basado en el campo del conocimiento de la Farmacología Veterinaria, que cumpla las funciones de obtener información contextualizada y centralizada de los fármacos de distribución nacional y regional en Medicina Veterinaria; el cual funcione desde el servidor del Laboratorio de Fisiología de la Universidad de los Llanos para la respectiva comunidad académica.

\section{MATERIALES Y MÉTODOS}

\section{Selección y organización de la información}

Se realizó la revisión de múltiples fuentes bibliográficas, en una primera instancia los libros insignia del proceso de enseñanza del área de la farmacología veterinaria a nivel del pregrado profesional, es decir Farmacología Clínica y Básica (Katzung, 2007), Goodman y Gilman, Las Bases Farmacológicas de la Terapéutica (Hardman, Goodman y Gilman, 2003), Manual De Farmacología Veterinaria (Plumb, 2008), Fundamentos de Farmacología Médica (Samaniego, 2005), Farmacología y Terapéutica Veterinaria (Adams, 2003), Farmacología y Terapéutica Veterinaria (Botana, 2002), Farmacología Humana (Flórez, 2005), Farmacología Veterinaria (Sumano y Ocampo, 2006), entre otros; además de diversas revistas científicas especializadas, entre las cuales se citan: Veterinary 
Pharmacology, Terapeuthics y la Revista Colombiana de Ciencias Pecuarias. Las bases de datos fueron otra fuente de información vanguardista sobre el tema, las principales consultadas fueron: ScienceDirect, Doyma, Scielo y EBSCO; también otros medios digitales se utilizaron, como lo fue la Farmacología interactiva UNAM, (http://www.labibliotecamedica.org/). A partir de estas fuentes bibliográficas se originó la matriz general de la información referente a la farmacología clínica y terapéutica veterinaria. En esta fase de recopilación de la información se realizó la extracción, procesamiento y retención de la información de los fármacos y medicamentos en libros de Excel ordenados de forma alfabética, para un total de 301 principios activos, que posteriormente entraron a la segunda etapa del proyecto: filtrado-selección de principios activos según disponibilidad en el contexto y usos profesionales.

\section{Construcción de la base de información del Farmacologix 1.0}

Se realizó el filtrado de los fármacos y medicamentos con mayor distribución comercial, a nivel nacional y regional en Medicina Veterinaria mediado por un proceso de selección intencional basado en la utilización de los principales fármacos trabajados en clínicas veterinarias y producciones animales de interés zootécnico. Adicional a esta selección se plasmó la clasificación de los medicamentos de acuerdo al Sistema de clasificación ATC (por su sigla en inglés The Anatomical, Therapeutic, Chemical Classification System) de sustancias farmacéuticas para uso humano, que incluye las siguientes variables: grupo farmacológico, principio activo (Saladrigas, 2004); adicionando: Farmacodinámica, farmacocinética, dosis, vía de administración y oferta comercial en Colombia.

Esta información se organizó en libros de Excel separados de acuerdo a la distribución sistemática del campo del conocimiento de la farmacología siguiendo los lineamientos propuestos por el Microdiseño del curso de farmacología de la Universidad de los Llanos (Jaramillo, 2009), así: Introducción a la Farmacología, Sistema Nervioso Central y Periférico, Sistema cardiovascular, Sistema renal, 
Sistema respiratorio, Sistema digestivo, Farmacología antibacteriana, antimicótica, Fármacos antineoplásicos, Farmacología de los antiparasitarios, antihistamínicos, antipiréticos, analgésicos, antiinflamatorios, antioxidantes; Farmacología reproductiva, Farmacología endocrina, Personajes, Glosario y Tablas de referencia de parámetros clínicos y paraclínicos de las especies animales de interés zootécnico.

\section{Programación extrema implícita en la creación del Farmacologix 1.0}

La Programación Extrema se basa en 12 principios básicos agrupados en cuatro categorías (Tomado y modificado de: Beck y Fowler, 2000), así:

1. Retroalimentación a escala fina: el principio de pruebas, proceso de planificación, el cliente en el sitio y programación en parejas.

2. Proceso continuo en lugar de por lotes, refactorización y entregas pequeñas.

3. Entendimiento compartido: diseño simple, metáfora, propiedad colectiva del código y estándar de codificación.

4. Proceso de desarrollo (Un desarrollo y los clientes finales)

Durante el diseño, desarrollo y pruebas del software, las cuales fueron la parte más importante en el proceso de la programación extrema, se revisó y mejoró de manera continua a lo largo del proyecto toda la información y su sistematización, según se van añadiendo funciones al mismo; cada vez que se implementó una parte de código, se realizó una prueba sencilla, y después se escribió el código para añadirlo a la programación final; por último se obtuvo un código simple y funcional de manera bastante rápida. El desarrollo del sistema de información se realizó bajo el entorno de software libre, en postgreSQL 8.4 siendo este un sistema de gestión de base de datos, publicado bajo la licencia BSD, éste es un sistema de administración relacional que archiva 
datos en tablas separadas, diferente de colocar todos los datos en un gran archivo; esto permite velocidad y flexibilidad, las tablas están conectadas por relaciones definidas que hacen posible combinar datos de diferentes tablas. En este caso el cliente "Farmacologix 1.0" fue creado en lenguaje Java versión 18, generando una interfaz que accede al cliente con la base de datos y permite crear, editar, eliminar, buscar y visualizar la información en el sistema

\section{Implementación del software para su manejo pedagógico-académico}

El sistema de registro de datos de escritorio Farmacologíx 1.0, funciona desde el servidor del Laboratorio de Fisiología de la Universidad de los Llanos; este sistema contextualiza y centraliza de manera eficiente información referente a los fármacos de distribución nacional y regional en Medicina Veterinaria; es así que los integrantes de la comunidad académica de la institución pueden acceder de forma libre y gratuita al servidor por conexión inalámbrica y consultar la base de datos creada. Sin duda alguna las repercusiones pedagógicas serán amplias y benéficas respecto al uso y aportes del software en el quehacer académico del Programa de Medicina Veterinaria y Zootecnia. La base de datos ofertada Farmacologix 1.0 se encuentra integrada por: 209 principios activos, 80 grupos farmacológicos, 12 sistemas o categorías de clasificación farmacológica, 24 personajes relevantes en el campo de la farmacología mundial, 24 términos en glosario, 68 revisiones bibliográficas y 3 tablas de referencia de parámetros clínicos y paraclínicos en animales de interés zootécnico.

El software cuenta con la interfaz principal, es decir, el sitio de información general del software, donde se encuentran los autores y vínculos principales de acceso al programa (Figura 1); el menú de búsqueda, opción donde se realiza los diferentes tipos de búsqueda disponibles, la búsqueda de medicamento, personaje, glosario, referencia bibliográfica, tablas de referencia (Figura. 2). El menú búsqueda de medicamento abre una ventana en la cual se selecciona el tipo de búsqueda para medicamentos, esta actividad hace referencia y permite realizar la búsqueda 
desde la categoría, grupo farmacológico, principio activo, oferta comercial, entre otras características del ingrediente activo (Figura. 3)

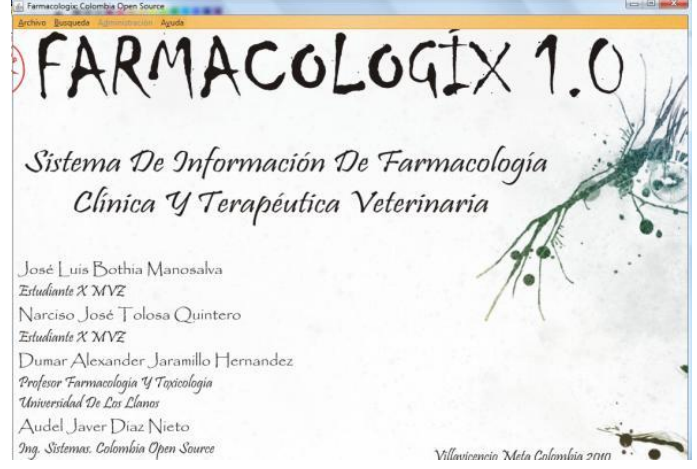

Figura 1. Interfaz principal, Farmacologíx 1.0.

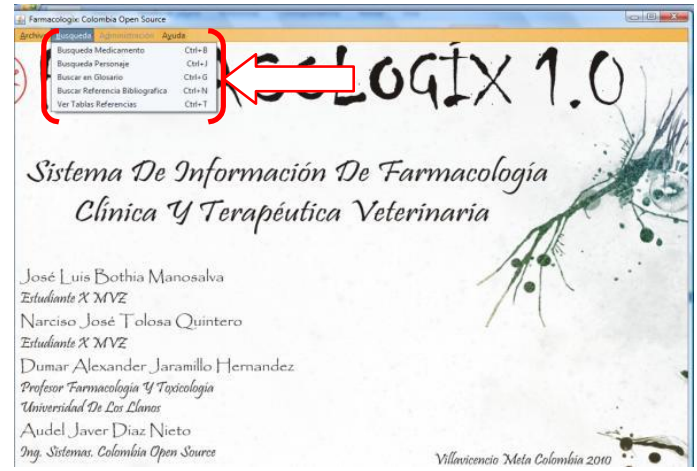

Figura 2. Menú búsqueda, Farmacologíx 1.0

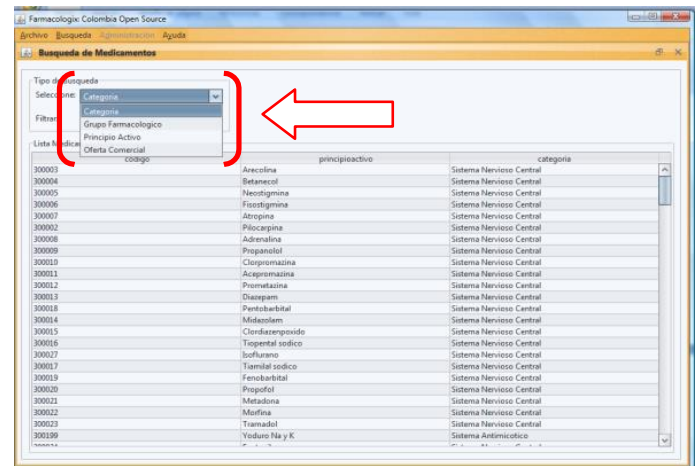

Figura 3. Búsqueda de medicamento, Selección tipo de búsqueda Farmacologíx. 
En la Figura 4 se puede observar toda la información del medicamento buscado, mostrado en los cuadros titulados con las siguientes variables: categoría, grupo farmacológico, principio activo, farmacodinámica, farmacocinética, indicaciones, dosis/administración, presentación comercial, tratamiento asociado a intoxicación y observaciones. En la Figura 5 se muestra la información referente al grupo farmacológico del medicamento buscado, también se incluye un diagrama de la acción general del grupo a nivel celular. En la Figura 6 se muestra la información referente a la categoría del medicamento buscado.

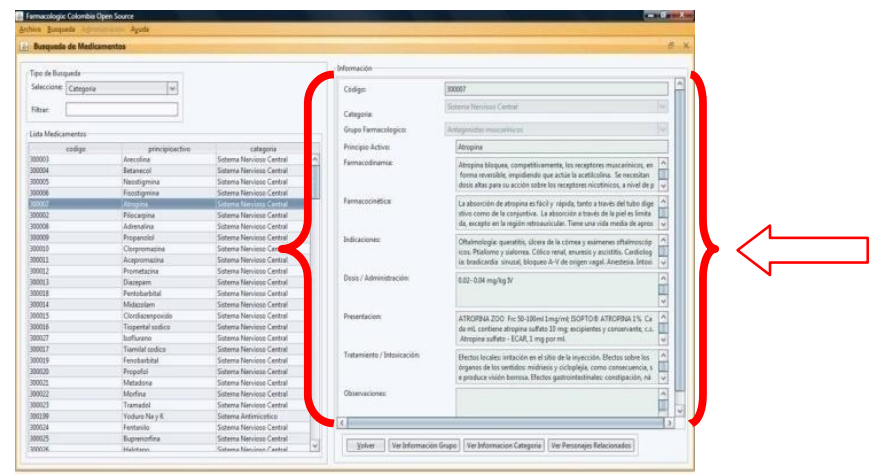

Figura 4. Información del medicamento buscado.

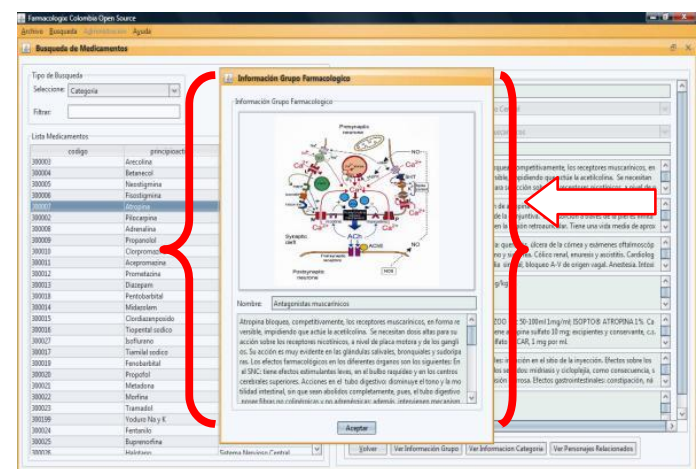

Figura 5. Información Grupo Farmacológico.

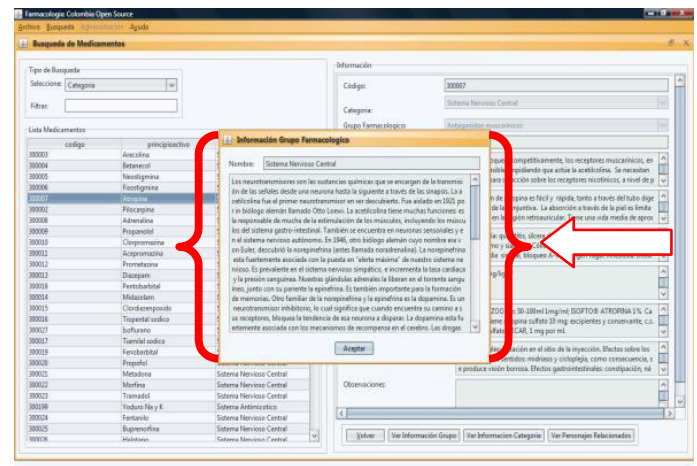

Figura 6. Información de la categoría. 
En el menú búsqueda de personaje: esta opción muestra los personajes más relevantes en la historia de la farmacología, especificando fotografía y su principal aporte.

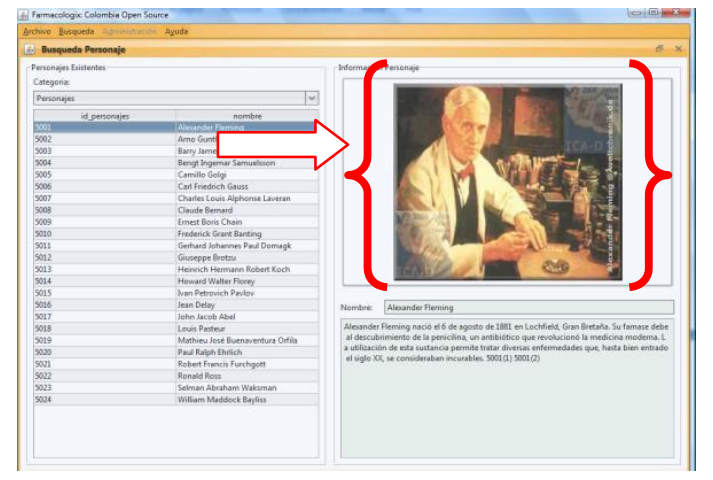

Figura 7. Búsqueda de personaje

\section{CONCLUSIÓN}

El software Farmacologíx 1.0. centraliza información confiable y relevante que permite tener mayor facilidad de aprendizaje y adquisición de competencias en el uso de los fármacos comercializados en la región Orinoquia y en Colombia; contribuyendo a mejorar las ayudas didácticas digitales a las cuales pueden acceder estudiantes y profesionales de la Medicina Veterinaria y Zootecnia para complementar los conocimientos generales y específicos del área de la Farmacología clínica y terapéutica veterinaria. Se recomienda mejorar la amplitud del servicio y distribución del software Farmacologix 1.0, incrementando nuevas herramientas y actualizaciones de la información de forma constante, para complementar la versión preliminar que se creó.

\section{REFERENCIAS BIBLIOGRÁFICAS}

1. Adams, H. R. Farmacología y terapéutica veterinaria. Segunda Edición. Editorial Acribia. USA. 2003

2. Adashi, E., Rock, J. A., Rosenwaks, Z. Reproductive Endocrinology, Surgery and Technology. Lippincott Raven. Philadelphia. 1995. 
3. APROVET. Vademecum Veterinario. Asociación Nacional de Laboratorios de Productos Veterinarios. Colombia. 2007.

4. Beck, K., Fowler, M. Planning Extreme Programming. Addison-Wesley Pub Co; 2000. Pp 160.

5. Botana L. M., Ocampo. Farmacología y terapéutica veterinaria. McGraw-Hill Interamericana. 2002.

6. ERMA. Laboratorios Farmacéuticos Veterinarios Erma. Colombia. 2004.

7. Katzung B. Farmacología Clínica y Básica. 10 ed. México DF: Editorial el Manual Moderno; pp. 714-5. 2007.

8. Florez, J. Farmacología humana. 4 ed. Editorial Masson. Barcelona 2005.

9. Hardman, Goodman, Gilman. Las Bases Farmacológicas de la Terapéutica. México DF: McGraw-Hill Interamericana; 2003.

10. Jaramillo D. A. Microdiseño de Farmacología y Toxicología Veterinaria. Líder del Grupo de Investigación en Farmacología Experimental y Medicina Interna - Élite. Universidad de los Llanos. 2009.

11. Majó, J. Nuevas tecnologías y educación. [online] Diciembre 2003. [Recuperado el 05 de marzo de 2010] en: http://www.uoc.edu/web/esp/articles/joan_majo.html.

12. PostgreSQL. PostgreSQL Global Development Group, versión 8.4 para Windows Xp 32 bits, actualizado 1 de julio de 2009, publicado bajo la licencia BSD (Berkeley Software Distribution).

13. Plumb. Manual De Farmacología Veterinaria. Editorial Intermedica. 2008.

14. Programa de Medicina Veterinaria y Zootecnia, Condiciones mínimas de calidad, Facultad de Ciencias Agropecuarias y Recursos Naturales, Universidad de los Llanos, 2004 
15. P.R. Vademecum. Vademecum. Colombia. 2009.

16. Saladrigas, M. V. El sistema de clasificación ATC de sustancias farmacéuticas para uso humano (The Anatomical, Therapeutic, Chemical Classification System) Panace@. Vol. V. 2004.

17. Samaniego, E., Fundamentos de Farmacología Médica. Quito: Editorial de la Universidad Central del Ecuador; 2005.

18. SCHERING-PLOUGH S.A. Vademecum Veterinario. Laboratorio farmacéutico Schering-Plough S.A. Colombia. 2004.

19. Sumano H., Ocampo L. Farmacología veterinaria, McGraw Hill Interamericana, 3를 ed. 1082 p. 2006.

20. VECOL. Vademecum veterinario. Empresa Colombiana de Productos Veterinarios, Bogotá. Colombia. 2003. 